\title{
Technology of AIRS/AMSU/ATMS satellite data processing
}

\author{
Egor Yu. Mordvin ${ }^{1,2}$, Anatoly A. Lagutin ${ }^{1,2}$ and Artemy I. Revyakin ${ }^{1}$ \\ ${ }^{1}$ Altai State University, Barnaul, Russia \\ ${ }^{2}$ Federal Research Center for Information and Computational Technologies, Novosibirsk, Russia
}

\begin{abstract}
The Atmospheric Infrared Sounder (AIRS) is a hyperspectral instrument with 2378 channels. It is a part of the Aqua space platform equipment. It registers outgoing longwave radiation in the IR-band from 3.74 to 15.4 microns. To correctly retrieve the atmospheric profiles in the presence of cloud structures, AIRS measurement processing scheme uses data from the 15-channel AMSU-A microwave instrument, which is also installed on Aqua. The paper proposes a technology for synthesizing the readings of AMSU-A, that failed in the fall of 2015, by using observations from the 22-channel radiometer of the Advanced Technology Microwave Sounder (ATMS) installed on Suomi-NPP and NOAA-20 satellites. These platforms were launched in 2011 and 2018, respectively. The transition between the coordinate grids of the two instruments was implemented by the "resample" software library, which transferred the radiance temperature values obtained by ATMS radiometer to the AMSU-A irregular measurement grid by means of a Gaussian function. The method was tested for the Aqua and Suomi-NPP neighboring orbits of 2015, when AMSU-A was still operating normally. It is established that the root-mean-square deviation of the radiance temperature values during the transferring of ATMS data to the AMSU-A coordinate grid does not exceed $1 \%$, and the correlation coefficient is 0.98 . Using the synthesized AMSU-A readings, reconstructions of the parameters of the atmosphere and the underlying surface were carried out. The analysis of the obtained results showed the suitability of the proposed method of replacing the microwave data from AMSU by the data from ATMS instruments. It should be noted that in the case of a rapidly changing atmosphere, for example, with a strong wind, the use of ATMS observations is possible only if the difference in the passage time of the two satellites does not exceed 10-15 minutes.
\end{abstract}

\section{Keywords}

AIRS, AMSU-A, ATMS, Aqua satellite, data reprojection.

\section{Introduction}

Currently, hyperspectral imaging in the IR range is an important source of satellite information that is used to assess climate changes [1], to monitor the state of the atmosphere and the underlying surface [2], and to measure the content of greenhouse gases [3]. The Atmospheric InfraRed Sounder (AIRS) is one of the six instruments of Aqua satellite, launched into lowEarth orbit in 2002 as part of the Earth Observing System (EOS) NASA project [4]. AIRS is a hyperspectral device with 2378 channels that register outgoing longwave radiation in the range

SDM-2021: All-Russian conference, August 24-27, 2021, Novosibirsk, Russia

Đion0210@gmail.com (E.Yu. Mordvin); lagutin@theory.asu.ru (A. A. Lagutin); artemy507@gmail.com

(A. I. Revyakin)

(D) 0000-0001-9327-100X (E. Yu. Mordvin); 0000-0002-1814-8041 (A. A. Lagutin); 0000-0001-5456-8900

(A. I. Revyakin)

(c) (1) $\odot 2021$ Copyright for this paper by its authors. Use permitted under Creative Commons License Attribution 4.0 International (CC BY 4.0).

[Den 
from 3.74 to 15.4 microns [5]. To correctly restore the geophysical parameters of the atmosphere and the underlying surface in the presence of cloud structures, the AIRS measurement processing scheme uses data from the 15-channel Advanced Microwave Sounding Unit (AMSU-A), also installed on Aqua. The joint use of the data of these two devices allowed us to restore the atmospheric parameters even with the $80 \%$ cloud cover of the observation zone [6].

In 2016, AMSU-A failed and its measurements were excluded from the AIRS data processing scheme. Despite the fact that the results of processing the current AIRS Only algorithm version 7 differ less from the results of AIRS/AMSU version 7 [7] than when comparing the previous algorithms versions 5 and 6 , the absence of microwave data significantly affects the correctness of reconstruction of the characteristics of the "atmosphere-underlying" surface system in areas covered by clouds [8]. In this regard, the joint use of AIRS measurements along with AMSU microwave data provides more accurate observations.

In October 2011, as a part of a sequence of Low Earth Orbiting satellite missions under the Joint Polar Satellite System (JPSS), the Suomi National Polar-orbiting Partnership (Suomi-NPP) platform, which carries a new generation of research instruments on board, was launched into a sun-synchronous orbit $[9,10]$. The hyperspectral imaging unit in the IR range is represented by the Cross-track Infrared Sounder (CrIS) [11] which works synchronously with the Advanced Technology Microwave Sounder (ATMS), similar to the AIRS/AMSU tandem. To extend the long-term series of AIRS/AMSU observations with new CrIS/ATMS results, an intercalibration procedure was performed [12]. It was necessary both for the readings of the instruments themselves $[12,13,14]$ and for the results of retrieving the characteristics of the atmosphere and underlying surface [15]. For Suomi-NPP instruments, this problem has a solution [12,13], since the observations of these satellite platforms have a temporary overlap of about five years. However, for the NOAA-20 satellite launched in 2018 as part of the Joint Polar Satellite System (JPSS) program [10], intercalibration with the results of AIRS/AMSU algorithm is not feasible.

The purpose of the study was to develop an approach for using the observations of ATMS installed on the Suomi-NPP and NOAA-20 in the data processing chain of AIRS/AMSU sounding suite.

\section{Instruments}

\subsection{AIRS/AMSU sounding suite}

Aqua space platform was launched on May 4, 2002 [4, 5] on a near-polar sun-synchronous orbit $704 \mathrm{~km}$ height. AIRS/AMSU sounding suite is based on two instruments.

AIRS scans the Earth's surface and atmosphere in a direction perpendicular to the satellite's velocity vector. The width of the scanning area is about $1600 \mathrm{~km}$. This instrument registers the intensity of outgoing thermal radiation in the ranges $3.74 \div 4.61$ microns $\left(2169 \div 2674 \mathrm{~cm}^{-1}\right)$, $6.20 \div 8.22$ microns $\left(1217 \div 1613 \mathrm{~cm}^{-1}\right), 8.8 \div 15.4$ microns $\left(649 \div 1136 \mathrm{~cm}^{-1}\right)$ with a high spectral resolution $\lambda / \Delta \lambda \sim 1200$. These spectral ranges include absorption zones important for the retrieval of atmospheric parameters: carbon dioxide (4.3 and 15 microns), water vapor (6.3 microns), methane (7.7 microns) and ozone (9.6 microns).

AMSU-A consists of two modules that include 15 channels. Module 2 (AMSU-A2) has 2 channels $(23.8$ and $31.4 \mathrm{GHz})$ that provide information about the emissivity of the surface, the content 
of water vapor and liquid water in the clouds. Module 1 (AMSU-A1) has 12 channels in the oxygen absorption band of $50-58 \mathrm{GHz}$, providing the possibility of primary temperature sensing, and also has 1 channel with a frequency of $89 \mathrm{GHz}$, providing information about the surface and humidity. The power supply of the AMSU-A2 was lost at 19:47 UTC on September 24, 2016.

\subsection{Microwave radiometer ATMS}

Suomi-NPP was launched on October 28, 2011. It moves along a polar sun-synchronous orbit, the height of which is approximately $824 \mathrm{~km}$, passing from the North Pole to the South Pole and back about 14 times per day [16]. Suomi-NPP research equipment consists of five instruments.

ATMS combines the capabilities of AMSU-A and the Microwave Humidity Sounder (MHS), which are currently installed on Polar Operational Environmental Satellites (POES/NOAA). However, compared to its predecessors, ATMS has: more channels (22 channels), a better spatial resolution, a wider range of received frequencies (from 21.8 to $183.3 \mathrm{GHz}$ ) [17]. Each channel is responsible for detecting the temperature of a certain atmospheric level. The characteristics of the ATMS channels are presented in Table 1. It can be clearly seen that the AMSU channels that have failed (indicated by an asterisk) have analogues in the detectors of ATMS. It should also be

\section{Table 1}

Characteristics of the ATMS channels.

\begin{tabular}{cccccc}
\hline No. & $\begin{array}{c}\text { Center of channel, } \\
\text { GHz }\end{array}$ & $\begin{array}{c}\text { Width of } \\
\text { channel, } \\
\text { GHz }\end{array}$ & $\begin{array}{c}\text { Solid } \\
\text { angle of view, } \\
\text { degree }\end{array}$ & $\begin{array}{c}\text { Measurement } \\
\text { error, } \\
\text { K }\end{array}$ & $\begin{array}{c}\text { Analogues } \\
\text { AMSU channel }\end{array}$ \\
\hline 1 & 23.8 & 0.27 & 5.2 & 1 & $1^{*}$ \\
2 & 31.4 & 0.18 & 5.2 & 1 & $2^{*}$ \\
3 & 50.3 & 0.18 & 2.2 & 0.75 & 3 \\
4 & 51.76 & 0.4 & 2.2 & 0.75 & - \\
5 & 52.8 & 0.4 & 2.2 & 0.75 & $4^{*}$ \\
6 & $53.596 \pm 0.115$ & 0.17 & 2.2 & 0.75 & $5^{*}$ \\
7 & 54.4 & 0.4 & 2.2 & 0.75 & $6^{*}$ \\
8 & 54.94 & 0.4 & 2.2 & 0.75 & $7^{*}$ \\
9 & 55.5 & 0.33 & 2.2 & 0.75 & 8 \\
10 & 57.290344 & 0.33 & 2.2 & 0.75 & $9^{*}$ \\
11 & $57.290344 \pm 0.217$ & 0.078 & 2.2 & 0.75 & 10 \\
12 & $57.290344 \pm 0.3222 \pm 0.048$ & 0.036 & 2.2 & 0.75 & 11 \\
13 & $57.290344 \pm 0.3222 \pm 0.022$ & 0.016 & 2.2 & 0.75 & 12 \\
14 & $57.290344 \pm 0.322 \pm \pm 0.010$ & 0.008 & 2.2 & 0.75 & 13 \\
15 & $57.290344 \pm 0.3222 \pm 0.0045$ & 0.003 & 2.2 & 0.75 & - \\
16 & 88.2 & 2 & 2.2 & 1 & - \\
17 & 165.5 & 3 & 1.1 & 1 & - \\
18 & $183.31 \pm 7$ & 2 & 1.1 & 1 & - \\
19 & $183.31 \pm 4.5$ & 2 & 1.1 & 1 & - \\
20 & $183.31 \pm 3$ & 1 & 1.1 & 1 & - \\
21 & $183.31 \pm 1.8$ & 1.1 & 1.1 & - \\
22 & $183.31 \pm 1$ & 0.5 & & 1 & - \\
\hline
\end{tabular}


noted that the width of the ATMS capture band is approximately $2300 \mathrm{~km}$.

After ATMS showed itself well as part of Suomi-NPP, it was decided to include the instrument in other satellite missions, such as: JPSS-1 (launched on November 18, 2017 [18]) and JPSS-2 (scheduled for launch in 2022 [18]). After the launch JPSS-1 was renamed to NOAA-20. The orbit configurations of NOAA-20 and Suomi-NPP are close to each other.

\section{Replacing of AMSU data}

\subsection{Criteria for replacing of AMSU data}

In the course of the work, the following criteria were formulated, according to which the AMSU data should be replaced with the results of observation of another microwave sounder (radiometer X):

1) the spectral channels of the radiometer X coincide with the AMSU channels;

2) the width of the scan area $X$ should be approximately equal to or greater than the AMSU parameters;

3) the spatial resolution of $X$ is comparable to the characteristics of AMSU;

4) the orbit configuration of the radiometer $X$ satellite platform should be similar to the Aqua trajectory;

5) the difference between the time of sensing the studied area performed by X and AMSU one should not exceed 30 minutes.

At the time of the study, the $\mathrm{X}$ instrument that fits all the criteria in a best manner is ATMS which is based on the Suomi-NPP and NOAA-20 space platforms [10, 18].

\subsection{Procedure of AMSU data replacing}

Since the scanning geometry, in particular, the pixel size and the width of the scanning area, of ATMS and AMSU instruments is different, in order to substitute the ATMS readings into the AIRS/AMSU algorithm, it is necessary to transfer this data to the AMSU coordinate system. The method chosen for the work should take into account the fact that the pixel sizes of AMSU and ATMS differs for the nadir and the edges of scanning zone. On the other hand, the surface whose radiation is recorded by satellite devices is not a plane but a geoid. The basis of the ATMS data transfer procedure was the Pyresample program interface.

Pyresample is a Python library designed for resampling geospatial images into various coordinate systems [19]. Its methods can work both with fixed spatial grids and with geolocated data in form of a capture band of a satellite instrument.

Pyresample includes several image resampling methods, which are shown below.

The nearest method where the value of the nearest point of a source raster is assigned to a target pixel;

The gauss method where the value of the target pixel $I$ is defined as an arithmetic weighted average, as shown in the following expression:

$$
I=\frac{\sum I_{i}^{\prime} w_{i}}{\sum w_{i}}
$$


where $I_{i}^{\prime}$ is the data of the original grid, and $w_{i}$ is the value of the weight function, which is determined according to:

$$
w=e^{\left(-D^{2} / \sigma^{2}\right)},
$$

where $D$ is the distance to each pixel that falls into the search area with a radius $R$, and $\sigma$ is a parameter that is not the standard deviation of the Gaussian.

The custom method where the value of the target pixel is determined similarly to the gauss method but the weight function is set by the user.

Using the functionality of the Pyresample library, a subroutine was implemented to transfer ATMS observations to the AMSU observation space. Working with ATMS data stored in HDF5 format files was implemented using the h5py interface. Reading the coordinates of AMSU pixels and writing the results of transferring ATMS data to HDF4 format files was performed using the pyhdf library. Operations with arrays were implemented using the numpy interface.

\subsection{Resampling result}

Testing of the ATMS data transfer methods was carried out using observations for 2015, when AMSU was functioning normally. For this purpose, such trajectories of the Aqua satellite were selected from the satellite archive of the ASU Space Monitoring Center, for which the difference between the observation time of AMSU and ATMS/Suomi-NPP did not exceed 15-20 minutes. In this case, the tracks (the projection of the satellite's trajectory on the Earth's surface) of the two space platforms have the smallest difference, and the parameters of the atmosphere and the underlying surface do not have time to change significantly. To cover the entire range of AMSU observations, the results of night and day surveys performed for all four seasons of the year were used. Table 2 shows the flight time of the Aqua and Suomi-NPP satellites over the territory of the Altai Region for test turns.

The calculations performed using the nearest and gauss methods with different parameter values $\sigma$ showed that the best result of the procedure for transferring ATMS data to the AMSU observation space is obtained for gauss $(\sigma=8000)$. It should be noted that in all experiments $R=45000 \mathrm{~m}$.

Figure 1 shows the ratio of AMSU observations to the results of the gauss method ( $\sigma=8000)$ performed for the entire test data set. It can be seen that there is a good agreement between the

\section{Table 2}

The time of the Aqua and Suomi-NPP flights over the territory of the Altai Region.

\begin{tabular}{ccc}
\hline Date & Aqua flight time & Suomi-NPP flight time \\
\hline 2015.01 .14 & $07: 04$ UTC & $07: 02$ UTC \\
2015.01 .16 & $20: 12$ UTC & $20: 10$ UTC \\
2015.03 .11 & $07: 43$ UTC & $07: 45$ UTC \\
2015.03 .11 & $20: 12$ UTC & $20: 33$ UTC \\
2015.07 .16 & $20: 32$ UTC & $20: 11$ UTC \\
2015.07 .19 & $07: 28$ UTC & $07: 05$ UTC \\
2015.10 .05 & $07: 40$ UTC & $07: 42$ UTC \\
2015.10 .07 & $20: 59$ UTC & $20: 56$ UTC \\
\hline
\end{tabular}




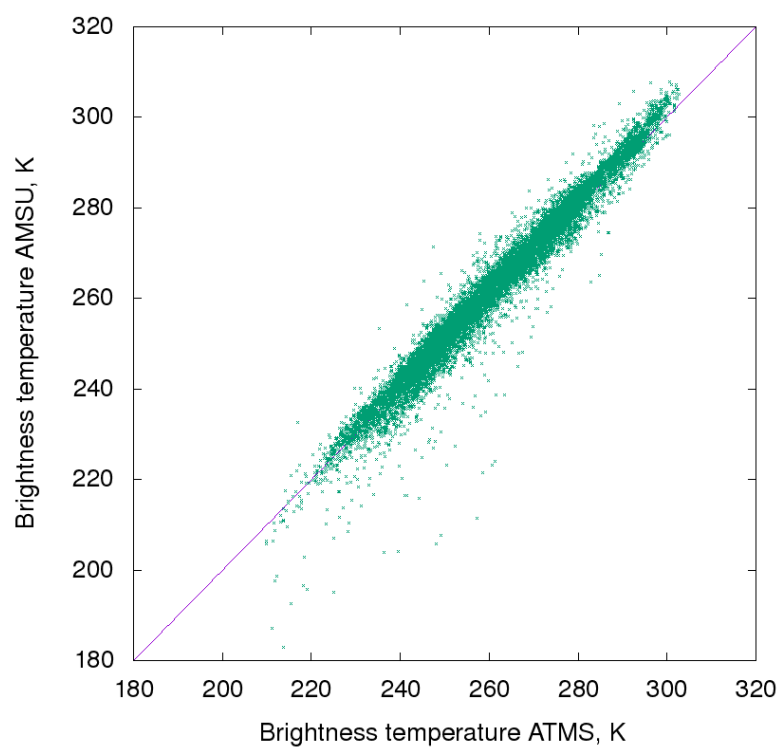

Figure 1: The ratio of observations in the 1st AMSU channel to the result of the gauss method $(\sigma=8000)$.

compared values. The resulting correlation coefficient $R^{2}$ for the test data set is 0.97 , and the standard deviation is $2.5 \mathrm{~K}$.

\section{AIRS/AMSU/ATMS computer complex}

\subsection{Computer complex for processing data from AIRS/AMSU/ATMS}

The development of the AIRS/AMSU/ATMS joint data processing computer complex was carried out on the basis of software created at the Space Monitoring Center of the Altai State University. It is based on the International MODIS/AIRS Processing Package (IMAPP) version 6 [20]. The program that controls the AIRS/AMSU data processing modules is implemented in the form of open source scripts. This allows one to make changes to the subroutine call order or to add new functions. The AIRS/AMSU/ATMS processing scheme implemented during the study is shown in Figure 2.

At the first stage, the "raw stream" received by the ASU satellite information receiving station is unpacked [3]. To do this, the RT-STPS (The Real-time Software Telemetry Processing System) package version 6.0 is used. At level 1a, geolocation is performed, which purpose is to assign geographical coordinates to each measurement made by the sensor. Level $1 \mathrm{~b}$ includes calibration of the readings of all instruments included in the sounding suite. The geolocation and calibration blocks are built on the basis of the computing package version 5.0.22. Retrieval of the atmosphere and underlying surface characteristics is performed at level 2. As shown in Figure 2, the ATMS data transfer routine to the AMSU coordinate system is called after performing step l1b. 


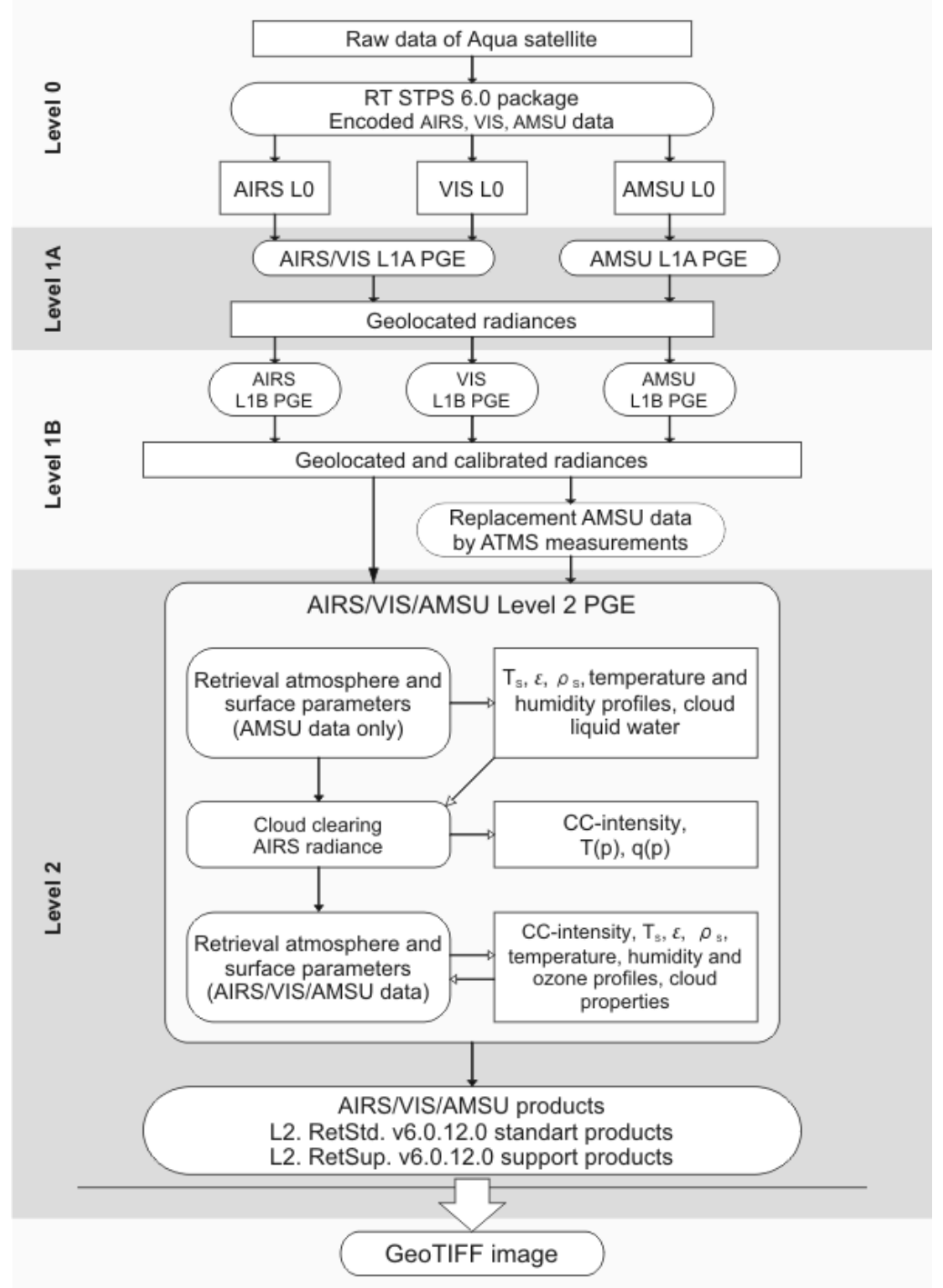

Figure 2: Joint AIRS/AMSU/AMSU data processing diagram.

\subsection{Verification of AIRS/AMSU/ATMS algorithm results}

Verification of the AIRS/AMSU/ATMS algorithm results was performed using observations for 2015, when AMSU was functioning normally. The test data set was compiled in such a way as to include the largest number of extreme states of the atmosphere and the underlying surface. For this purpose, the results of night and day surveys performed in different climatic seasons of the year were selected. Just as for the verification of $11 \mathrm{~b}$ level products, Aqua tracks were selected from the digital archive of the ASU Space Monitoring Center for which the difference with the Suomi-NPP trajectory did not exceed 15-20 minutes.

Figure 3 shows the temperature of the near-surface air at 20:16 UTC on January 16, 2015, ob- 


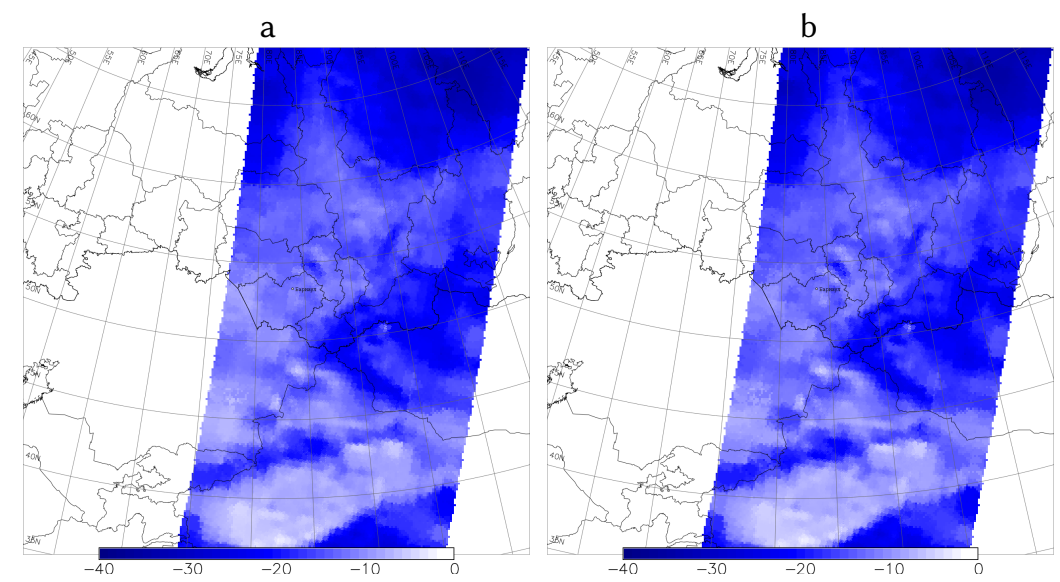

Figure 3: The near-surface air temperature according to the AIRS/AMSU version 6 algorithm (a) and the results of the AIRS/AMSU/ATMS joint processing (b) at 20:16 UTC on January 16, 2015.

tained according to the AIRS/AMSU version 6 algorithm (a), and the results of joint processing of AIRS/AMSU/ATMS observations (b). The figure shows that the estimates of AIRS/AMSU/ATMS agree quite well with the original results of the AIRS/AMSU algorithm version 6. A similar result was obtained for the observations made at 07:20 UTC on July 19, 2015, which are shown in Figure 4.

Figure 5 shows the ratio of the data on the surface air temperature contained in the standard AIRS/AMSU product version 6 and the estimates obtained as a result of substituting ATMS observations for the entire test data set. It can be seen that the compared values agree well.

To numerically estimate the difference between the reference and new data, the standard deviation and the correlation coefficient were obtained $R^{2}$, which are equal to $0.8 \mathrm{~K}$ and 0.98 , respectively. Despite the fact that the verification was performed only for eight satellite scenes, the standard deviation has a value comparable to the measurement error of the AIRS/AMSU

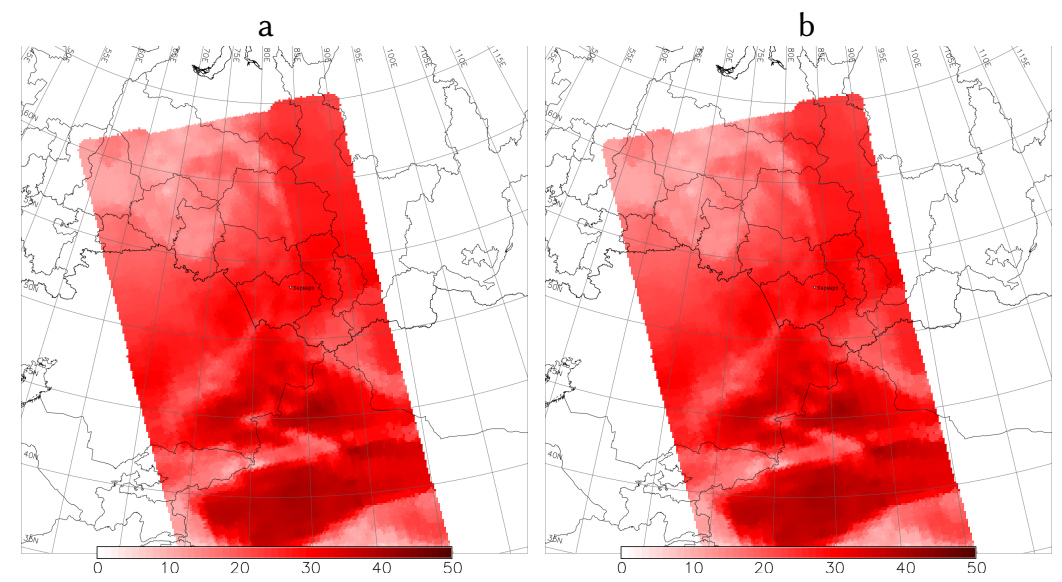

Figure 4: The near-surface air temperature according to the AIRS/AMSU version 6 algorithm (a) and the results of the AIRS/AMSU/ATMS joint processing (b) at 07:20 UTC on July 19, 2015. 


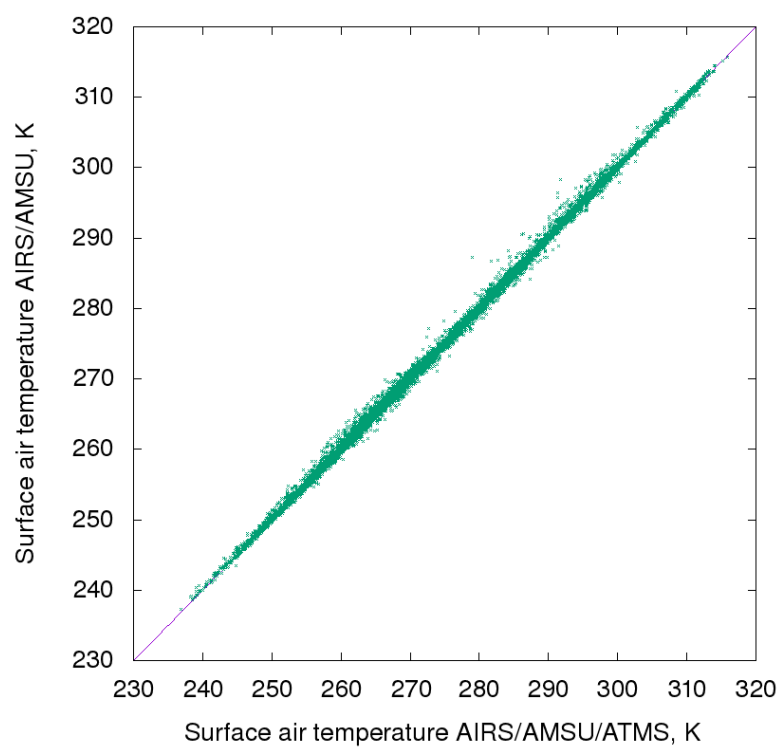

Figure 5: The ratio of the surface air temperature obtained according to the AIRS/AMSU version 6 algorithm and the results of joint AIRS/AMSU/ATMS processing

version 6 algorithm. This allows us to conclude that the developed method is applicable for processing AIRS/AMSU data received after September 2016. This approach can be used to extend a number of AIRS/AMSU observations, as well as to perform intercalibration of next-generation instrument data.

\section{Conclusion}

The work was devoted to the development of an approach for using the observations of ATMS instrument, installed on Suomi-NPP and NOAA-20 satellites, in the data processing chain of AIRS/AMSU sounding suite based on Aqua spaceborne platform. In the course of the study, the following results were obtained:

1. A subroutine has been developed to transfer the ATMS readings into the AMSU observation space. It is established that using the Gaussian function with the parameters: $R=45000 \mathrm{~km}, \sigma=8000$, the mean square deviation between the AMSU data and the transfer results is $2.5 \mathrm{~K}$, and the correlation coefficient $R^{2}$ is 0.98 .

2. A computer complex has been developed for processing AIRS/AMSU data using observations of ATMS.

3. The results of the AIRS/AMSU/ATMS joint data processing software package were verified using observations obtained in 2015, when AMSU was operating normally. It is shown that for the TSurfAir (Temperature of the Surface Air) product, the standard deviation and the correlation coefficient $R^{2}$ are equal to $0.8 \mathrm{~K}$ and 0.98 , respectively. 


\section{References}

[1] P. R. Shukla, Climate Change and Land: an IPCC special report on climate change, desertification, land degradation, sustainable land management, food security, and greenhouse gas fluxes in terrestrial ecosystems. IPCC, 2019. URL: http://www.ipcc.ch/site/assets/uploads/ 2019/11/SRCCL-Full-Report-Compiled-191128.pdf.

[2] A. A. Lagutin, Y. A. Nikulin, A. A. Lagutin et al., Mathematical technologies of operational regional satellite monitoring of atmospheric characteristics and underlying surface. Part 2. AIRS, Computing technologies. 12(5) (2007) 78-102. (in Russian).

[3] E. Y. Mordvin, A. A. Lagutin, Methane in the atmosphere of Western Siberia, Azbuka, Barnaul, 2016. (in Russian).

[4] C. L. Parkinson, Aqua: An Earth-observing satellite mission to examine water and other climate variables, IEEE Trans. Geosci. Remote Sens. 41 (2003) 173-183. doi:10.1109/ TGRS. 2002.808319.

[5] H. H. Aumann, M. T. Chahine, C. Gautier et al., AIRS/AMSU/HSB on the Aqua mission: Design, science objectives, data products, and processing systems, IEEE Trans. Geosci. Remote Sens. 41 (2003) 253-264. doi:10.1109/TGRS . 2002 . 808356.

[6] J. Susskind, J. M. Blaisdell, L. Iredell et al., Improved temperature sounding and quality control methodology using AIRS/AMSU data: The AIRS science team version 5 retrieval algorithm, IEEE Trans. Geosci. Remote Sens. 49 (2011) 883-907. doi:10 . 1109/TGRS . 2010 . 2070508.

[7] J. Susskind, AIRS-Team Retrieval For Core Products and Geophysical Parameters: Versions 6 and 7 Level 2, 2020. URL: https://docserver.gesdisc.eosdis.nasa.gov/public/project/AIRS/ L2_ATBD.pdf.

[8] J. Susskind, C. D. Barnet, J. M. Blaisdell, Retrieval of atmospheric and surface parameters from AIRS/AMSU/HSB data in the presence of clouds, IEEE Trans. Geosci. Remote Sens. 41 (2003) 390-409. doi:doi:10 .1109/TGRS . 2002.808236.

[9] C. Cao, F. J. De Luccia, X. Xiong et al., Early On-Orbit Performance of the Visible Infrared Imaging Radiometer Suite Onboard the Suomi National Polar-Orbiting Partnership (S-NPP) Satellite, IEEE Trans. Geosci. Remote Sens. 52 (2014) 1142-1156.

[10] M. D. Goldberg, H. Kylcoyne, A. Cikanek et al., Joint Polar Satellite System: The United States next generation civilian polar-orbiting environmental satellite system, J. Geophys. Res. Atmos. 118 (2013) 13463-13475.

[11] C. Cao, Y. Chen, X. Jin et al., Cross Track Infrared Sounder (CrIS) Sensor Data Record (SDR) User's Guide Version 1.1, 2017. URL: https://www.star.nesdis.noaa.gov/jpss/documents/ UserGuides/.

[12] G. Chander, T. J. Hewison, N. Fox et al., Overview of Intercalibration of Satellite Instruments, IEEE Trans. Geosci. Remote Sens. 51 (2013) 1056-1080.

[13] L. L. Strow, C. Hepplewhite, H. Motteler, Full Spectrum Intercalibration of AIRS and CrIS, 2014. URL: https://airs.jpl.nasa.gov/system/presentations/files/107_sno_strow.pdf.

[14] L. L. Strow, H. Motteler, C. Hepplewhite et al., Intercalibration of AIRS and CrIS via SNOs, PDFs, and Large Random Samplings, 2017. URL: https://airs.jpl.nasa.gov/system/ presentations/files/394_IntercalibrationStrow.pdf.

[15] L. L. Strow, S. D.-S. Machado, H. Motteler et al., Inter-Calibration of AIRS with IASI 
and CrIS, 2012. URL: https://digitalcommons.usu.edu/cgi/viewcontent.cgi?article=1108\& context=calcon.

[16] Suomi National Polar-orbiting Partnership, (Lastaccessed July 25, 2021). URL: http://www. jpss.noaa.gov/mission_and_instruments.html.

[17] E. Kim, C.-H. J. Lyu, K. Anderson et al., S-NPP ATMS instrument prelaunch and on-orbit performance evaluation, J. Geophys. Res. Atmos. 119 (2014) 5653-5670.

[18] Joint polar satellite system, (Lastaccessed July 25, 2021). URL: http://www.jpss.noaa.gov/ mission_and_instruments.html.

[19] Pyresample, (Lastaccessed July 25, 2021). URL: http://pyresample.readthedocs.io/.

[20] International MODIS/AIRS Processing Package (IMAPP), 2021. URL: http://cimss.ssec.wisc. edu/imapp/. 